\title{
Erratum to: Can Anyone, and Should Anyone, Lead Innovation?
}

\section{Erratum to: \\ Chapter 2 in: C.L. Spiro, From Bench to Boardroom, https://doi.org/10.1007/978-3-319-64155-3_2}

After publication of the original version of this book, in Chapter 2 on Page 11, the chapter opening sentence has been revised as below:

Since I've written a book about it, you can probably guess where I stand on this question of whether you can, and should, lead innovation: Yes you can, and yes you should!

The updated online version of this chapter can be found at https://doi.org/10.1007/978-3-319-64155-3_2 